\title{
TÉCNICAS E INDICADORES PARA LA EVALUACIÓN DE PORTALES EDUCATIVOS EN INTERNET
}

AQUILES BEDRIÑANA ASCARZA*

E-mail: aquilesetelefonica.net.pe

\begin{abstract}
RESUMEN
Dadas algunas ideas preliminares de diferencias entre las páginas web y los portales educativos., podemos decir que todo portal es una página, pero no todo web es un portal. Este artículo presenta una ficha para catalogación y portales educativos cuyo autor es Pere Marqués, UAB, 2001.

También es necesario hacer una apreciación crítica de si la actual página web de la Universidad Nacional Mayor de San Marcos tiene la categoría de un portal educativo; con estas bases se diseñará el portal educativo de la Facultad de Ciencias Administrativas.
\end{abstract}

Palabras clave: Páginas web, portal educativo.

\section{ABS TRACT}

It given some preliminary ideas of differences between the web pages and the educational entries. Every entry is a web page but not every web is an entry. This article presents a slip for cataloguing and educational entries whose author is Pere Marqués, UAB, 2001.

Also he is necessary to do an appreciation criticizes if the present web page of the Greater National University of San Marcus has the category of an educational entry with these bases will be designed the educational entry of the Faculty of Administrative Sciences.

Keywords: Web pages, educational enters.

\footnotetext{
Doctor en Educación, Profesor Principal, Docente Investigador de la Unidad de Investigación y Decano de la Facultad de Ciencias Administrativas de la UNMSM. Profesor de Post Grado en la UNMSM.
} 


\section{INTRODUCCIÓN}

El objetivo del presente trabajo es estudiar los criterios de evaluación y clasificación, al menos de una forma somera, de los portales educativos que podamos encontrar en nuestro medio y que nos permitan presentar propuestas para el diseño del Portal Educativo de la Facultad de Ciencias Administrativas de la Universidad Nacional Mayor de San Marcos.

Los portales, frente a las páginas web o sitios web, tienen características diferenciadoras, de forma que si somos capaces de delimitarlas y ponderar el grado de cumplimento de estas, estaremos en condiciones de aproximar una respuesta a cuestiones tales como si estamos realmente ante un portal o es otra cosa, qué tipo de portal es, y dentro de su grupo de similares, qué aspectos están más o menos conseguidos respecto a los demás. Uno de los aspectos a evaluar con mayor detalle será el buscador (o motor de búsqueda) del portal, y dentro de ello incidiremos con mayor profundidad en lo referido a cuestiones de precisión y exhaustividad.

\section{DEFINICIÓN DE PORTAL}

Se puede ofrecer una primera definición básica diciendo que un portal es un punto de entrada a Internet donde se organizan contenidos, ayudando al usuario y concentrando servicios y productos, de forma que le permitan a éste hacer cuanto necesite hacer en Internet a diario, o al menos que pueda encontrar allí todo cuanto utiliza cotidianamente, sin necesidad de salir de dicho sitio.

Profundizando en la definición de portal es preciso matizar un poco más esa primera acepción básica. Si se limitara la definición a decir que un portal es «un punto de acceso a Internet» se estaría aportando muy poco, pues casi cualquier página personal o home-page de un sitio web (http://www. unmsm.edu.pe por ejemplo), podría ser un punto de acceso a Internet, pero no un portal propiamente hablando.

Un portal es, en todos los casos, un sitio web (website), una página web, pero no viceversa. No todas las páginas web ni todo sitio web sería un portal. Página web y sitio web son conceptos con una clara relación de sinonimia, aunque en realidad es una relación partitiva, ya que un sitio web está formado por una dirección y por un conjunto de páginas. Se pueden matizar ambos conceptos afirmando que un «sitio web» está compuesto por una o más páginas, pero sin que necesariamente esté implícita la idea de organización de información con criterios rigurosos.

Aunque hemos encontrado bastantes definiciones y descripciones del término portal que profundizan en este concepto, consideramos interesante utilizar la lista de características fundamentales de un portal que sugiere David Morrison (técnico especialista de la compañía Lotus), que pueden ayudar a reconocer un portal frente a otro tipo de páginas web. Para ello, Morrison usa las iniciales del término portal:

Personalización para usuarios finales.

organización del escritorio.

Recursos informativos divididos y organizados.

Trayectoria o seguimiento de las actividades de los usuarios (tracking).

Acceso a base de datos.

Localización de gente o cosas importantes.

El aspecto fundamental que subyace en estas características está relacionado de forma directa con la personalización. Mejorando la personalización del portal del usuario, y consiguiendo así un «portal a medida», se podrían reducir problemas cruciales como la dificultad de navegación y localización de información relevante, o la dificultad para la interacción del portal con las actividades cotidianas, especialmente en situaciones de alta movilidad del usuario.

Un buen portal debería identificar al usuario que ya ha visitado el portal y permitirle personalizar tanto la interfase (la visualización de la pantalla o «escritorio») como los contenidos, de forma que aquellos datos que se muestren estuvieran relacionados al máximo con él, ya fuere la cotización de sus acciones o el tipo de cambio de una moneda determinada, un calendario con fechas relevantes (el cumpleaños de su pareja, un examen, una reunión importante, etc.) Estos datos habrán de ser los adecuados a su perfil o rol dentro de una institución, adaptados a sus preferencias y necesidades, lo que hará que el aspecto formal y la distribución de contenidos de un portal que utilizan dos usuarios podrá diferir por completo, aún tratándose en realidad del mismo para ambos. 


\section{PORTALES EDUCATIVOS}

Los portales educativos son espacios web que ofrecen múltiples servicios a los miembros de la comunidad educativa (profesores, alumnos, gestores de centros y familias), tales como información, instrumentos para la búsqueda de datos, recursos didácticos, herramientas para la comunicación interpersonal, formación, asesoramiento, entretenimiento, etc. La evaluación de un portal educativo es un trabajo multidisciplinario de tecnólogos, pedagogos y psicólogos que rebasan los alcances del presente artículo.

\subsection{APORTACIONES DE LOS PORTALES EDUCA- TIVOS}

Las ventajas que proporcionan los portales educativos a sus destinatarios (profesores, estudiantes, familias) se derivan de los servicios que ofrecen, y del hecho de estar accesibles desde Internet en cualquier momento y lugar. Podemos destacar las siguientes:

- Proporcionar información de todo tipo a profesores, estudiantes y padres, así como instrumentos para realizar búsquedas en Internet.

- Proporcionar recursos didácticos de todo tipo, gratuitos y utilizables directamente desde Internet (materiales didácticos on-line) o desde los ordenadores (tras «bajar» una copia desde el portal).

- Contribuir a la formación del profesorado, mediante informaciones diversas y cursos de actualización de conocimientos.

- Asesorar a los profesores. En algunos casos los portales tienen espacios de asesoramiento sobre diversos temas: didáctica, informática, leyes, etc.

- Abrir canales de comunicación (foros, chats, listas...) entre profesores, estudiantes, instituciones y empresas de todo el mundo. A través de ellos se comparten ideas y materiales, se debaten temas, se consultan dudas.

- Proporcionar instrumentos para la comunicación: correo electrónico, chats, espacios para alojar páginas web.

\subsection{SUGERENCIAS PARA SU USO}

No obstante, aunque los portales educativos ofrecen muchos servicios a profesores, estudiantes y familias, también están llenos de elementos distractores que fácilmente pueden hacer que sus visitantes empiecen una navegación sin rumbo fijo que les haga perder mucho tiempo.

Situados en la perspectiva del profesor, es conveniente que este conozca los portales educativos a su alcance (labor que puede hacer poco a poco aprovechando «tiempos remanentes» que muchas veces se forman en su horario, tales como tiempos previos y posteriores a reuniones, fin anticipado de una actividad, etc.). Es importante que los profesores conozcan los servicios que puede obtener de cada portal, vean cuáles son de su interés (hay que procurar reducir al máximo estos portales de referencia) y determinen el portal que realmente le conviene visitar cuando necesite alguno de estos servicios.

\section{PLANTILLA DE EVALUACIÓN}

Al evaluar estos portales se tendrán en cuenta los servicios que ofrecen así como múltiples aspectos técnicos, ergonómicos, pedagógicos y funcionales. Todo ello queda recogido en la correspondiente plantilla para la catalogación y evaluación de portales educativos.

La plantilla para la catalogación y evaluación de portales educativos presenta tres espacios bien determinados, según Pere Marqués:

- Identificación. La primera parte de la ficha recoge datos básicos para la identificación del producto: título, URL, autores, editores, destinatarios, etc.

- Servicios que proporciona. Un listado de servicios que proporciona el portal a sus destinatarios.

- Evaluación. Presenta una tabla con determinados aspectos funcionales, técnicos y pedagógicos que deben evaluarse a partir de la consideración de una escala de 4 valores: Baja (si la característica que se evalúa está mal); Correcta (si este aspecto está bien, correcto); Alta (si este aspecto está muy bien) y Excelente (si resulta casi inmejorable en este aspecto) .

- Observaciones y evaluación global. Donde además de establecer una valoración global para el portal, se recogen determinadas observaciones complementarias que quiera hacer el evaluador. 
FICHA PARA LA CATALOGACIÓN Y EVALUACIÓN DE PORTALES EDUCATIVOS

(1) Pere Marqués-UAB, 2001

NOMBRE DE LA PÁGINA Programa Huascarán

DIRECCIÓN URL www.huascaran.gob.pe

AUTORES / EDITORES: Ministerio de Educación.

PATROCINADORES

PRINCIPALES DESTINATARIOS: ALUMNOS - PROFESORES - FAMILIAS

LIBRE ACCESO: SÍ-NO -III-INCLUYE PUBLICIDAD: SÍ NO -III-ACCESO WAP: SÍ NO

PRINCIPALES SERVICIOS QUE PROPORCIONA

Informativos $I$

Instrumentos para la

búsqueda de

información

- Noticias

- Agenda

- Acceso a "mass media": radio, TV, prensa...

- Legislación sobre temas educativos

- Diseños curriculares base, programas de las materias y asignaturas

- Información sobre recursos educativos: libros, software, vídeos...

- Selección comentada de páginas web de interés educativo.

- Listado de centros de recursos y bibliotecas (catálogo de servicios...)

- Listado de centros, recopilación de la oferta educativa por ciudades, barrios..

- Información concentrada de las ofertas de formación permanente

- Ofertas: viajes, productos diversos...

Formativos para el

- Buscadores de Internet, metabuscadores, índices temáticos...

profesorado

- Recopilación de experiencias educativas, buenas prácticas, didáctica.

- Recopilación de consejos y reflexiones sobre el uso de las NTIC en la escuela

- Cursos diversos, actividades de aprendizaje on-line

Recursos didácticos $\quad$-Recursos educativos utilizables gratuitamente

- Materiales diversos para estudiantes: apuntes, trabajos, exámenes..

- Diccionario / enciclopedia básica / biografías

- Atlas y mapas de todo el mundo

- Manuales NTIC

Asesoramiento

- Asesoramiento didáctico

- Asesoramiento informático

- Asesoramiento legal

- Asesoramiento sobre la educación de los hijos y gestión de la familia

- "El profesor particular en casa"

Canales de

- Bolsa de trabajo

comunicación

- Anuncios: segunda mano.

Instrumentos para la

comunicación

- Acceso a foros: listas, news, chats...

- Servicios de correo electrónico: buzón de correo

- Servicio de web mail

- Espacios web para crear páginas web

- Creación de foros

Entretenimiento

- Traductor on-line

- Juegos on-line

- Postales, felicitaciones, música...

(marcar con una X) 


\begin{tabular}{|c|c|c|c|c|}
\hline \multicolumn{5}{|c|}{ ASPECTOS FUNCIONALES. UTILIDAD (marcar con una $X$, donde proceda, la valoración) } \\
\hline & EXCELENTE & ALTA & CORRECTA & BAJA \\
\hline \multicolumn{5}{|l|}{ Relevancia, interés de los servicios que ofrece....................... } \\
\hline \multicolumn{5}{|l|}{ Facilidad de uso e instalación de los visualizadores............... } \\
\hline \multicolumn{5}{|l|}{ Carácter multilingüe, al menos algunos apartados principales } \\
\hline \multicolumn{5}{|l|}{ Múltiples enlaces externos........................................... } \\
\hline \multicolumn{5}{|l|}{ Canales de comunicación bidireccional ............................. } \\
\hline \multicolumn{5}{|l|}{ Servicios de apoyo on-line .............................................. } \\
\hline \multicolumn{5}{|l|}{ Créditos: fecha de la actualización, autores, patrocinadores ....... } \\
\hline \multicolumn{5}{|c|}{\begin{tabular}{|l|l} 
Ausencia o poca presencia de publicidad....................... & \\
\end{tabular}} \\
\hline \multicolumn{5}{|c|}{ ASPECTOS TÉCNICOS Y ERGONÓMICOS } \\
\hline & EXCELENTE & ALTA & CORRECTA & BAJA \\
\hline \multicolumn{5}{|l|}{ Entorno audiovisual: presentación, pantallas, sonido, letra....... } \\
\hline \multicolumn{5}{|l|}{ Elementos multimedia: calidad, cantidad......................... } \\
\hline \multicolumn{5}{|l|}{ Calidad y estructuración de los contenidos ..................... } \\
\hline \multicolumn{5}{|l|}{ Estructura y navegación por las actividades, metáforas } \\
\hline \multicolumn{5}{|l|}{ 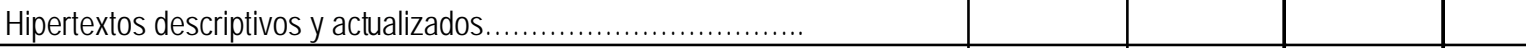 } \\
\hline \multicolumn{5}{|l|}{ Ejecución fiable, velocidad y visualización adecuada ....... } \\
\hline \multicolumn{5}{|l|}{ Originalidad y uso de tecnología avanzada.................... } \\
\hline \multicolumn{5}{|c|}{ ASPECTOS PEDAGÓGICOS } \\
\hline & EXCELENTE & ALTA & CORRECTA & BAJA \\
\hline \multicolumn{5}{|l|}{ Atractivo, capacidad de motivación, interés................................... } \\
\hline \multicolumn{5}{|l|}{ Adecuación a los destinatarios de los contenidos, actividades. } \\
\hline \multicolumn{5}{|l|}{ Recursos para buscar y procesar datos....................... } \\
\hline \multicolumn{5}{|l|}{ Potencialidad de los recursos didácticos: síntesis, resumen.. } \\
\hline \multicolumn{5}{|c|}{$\begin{array}{l}\text { OBSERVACIONES } \\
\end{array}$} \\
\hline \multicolumn{5}{|l|}{$\begin{array}{l}\text { Aspectos más positivos del portal: } \\
\text { Aspectos más negativos del portal: } \\
\text { Otras observaciones } \\
\text { - }\end{array}$} \\
\hline \multicolumn{5}{|c|}{ VALORACIÓN GLOBAL DEL PORTAL } \\
\hline & EXCELENTE & ALTA & CORRECTA & BAJA \\
\hline \multicolumn{5}{|l|}{ Los servicios que ofrece (es completo)............................. } \\
\hline \multicolumn{5}{|l|}{ 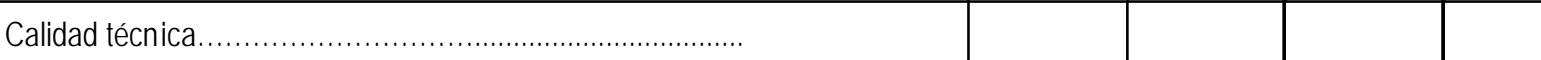 } \\
\hline Funcionalidad, utilidad para sus usuarios............................ & & & & \\
\hline
\end{tabular}




\section{APRECIACIÓN CRÍTICA DEL PORTAL EDUCATIVO DE LA UNIVERSIDAD NACIONAL MAYOR DE SAN MARCOS}

Aplicando los criterios de evaluación pedagógica y los indicadores anteriormente descritos, podemos decir que el portal educativo de la Universidad puede considerarse como un prototipo que tiene que seguir desarrollándose para alcanzar la categoría de un verdadero portal educativo.

El portal de la universidad tiene muchos vacíos y deficiencias que pueden superarse con un trabajo en equipo de docentes especializados y el apoyo de expertos en el tema.

Las debilidades del portal en referencia pueden ser:

- Carece de manuales de NTIC para docentes.

- Carece de buscadores y/o metabuscadores.

- No tiene asesoramiento didáctico.

- Carece de web mail.

- No cuenta con espacios de servicios: avisos clasificados, bolsa de trabajo, etc.

- Falta estandarizar formatos.

- Acceso a medios de comunicación masiva, radio, televisión, prensa escrita.

- Legislación sobre temas educativos.

Considero importante que se realice un panel de expertos para evaluar el portal y todas las herramientas educativos que viene usando la Universidad.

Nuestra política es analizar y evaluar los valores que aparecen en el portal y las herramientas educativas, para ello necesitamos un instrumento adecuado, un instrumento sensible que sea capaz de sacar a la luz los fundamentos axiológicos que se proponen, a veces claramente, otras de modo implícito en los programas educativos.

Para ello, tenemos que atender a la información que se presenta haciendo uso del lenguaje (tanto hablado como escrito), la imagen gráfica, la música y otros sonidos y/o ruidos, y ello considerando cada elemento por separado, como las combinaciones posibles entre ellos.

Los instrumentos habitualmente empleados para la evaluación del portal educativo son fundamentalmente de tipo técnico (presentación, software y hardware necesarios, destinatarios, etc.), de tipo pedagógico (objetivos, contenidos, actividades y evaluación que se trabajan) o de aspectos funcionales (ventajas respecto a otros medios, cómo, para qué y cuándo utilizarlos, etc. $)^{1}$. Sin embargo el instrumento que necesitamos debe centrarse en la evaluación axiológica; por esa razón entendimos que la investigación e innovación es este campo es muy importante.

\section{NOT A}

${ }^{1}$ Marqués, 1999.

\section{BIBLIOGRAF ÍA}

Adell, J. «Redes y Educación». En: J. de Pablos y J. Jiménez (Eds.) Nuevas Tecnologías. Comunicación Audiovisual y Educación. Barcelona, CEdecs Psicopedagogía, pp. 177$212,1998$.

Bitter, G. Y Pierson, M. Using technology in the classroom. USA, Allyn and Bacon, 2002.

Burbules, N. y Torres, C. A. Globalización y educación. En: Revista de Educación. Número Extraordinario, 2001, pp. 13-28,

Castells, M. La era de la información. En: Economía, sociedad y cultura. I, II, III. Madrid, Alianza, 1997.

Harmon, S. W. The five levels of Web use in education: Factors to consider in planning an online course. En: Educational Technology. 36 (6), 1999, pp. 28-32.

Lowther, D. L.; Jones, M. G. Y Plants, R. T. Preparing Tomorrow's teachers to use web-based Education. In: A. B. (Ed.) Instructional and cognitive impacts of webbased education. USA, Idea Group Publishing, , 2000, pp. 129-146.

Manzano, R. J. A different kind of classroom: teaching with dimensions of learning. Alexandría, Association for supervision and Curriculum Development, 1992.

Alonso, A. y Gallego, D. Madrid, Editores Multimedia, UNED, 1997.

De Benito Crosetti, B. Herramientas para la creación, distribución y gestión de cursos a través de internet. En: EDUTEC. N. ${ }^{\circ} 12$. Junio, 2000.

Bethoney, H. Anytime/Anyplace learning. ShootOut puts Web-based learning management systems to the test. PC Week Labs, 15 november, 1999. 
Bonk, C. J. y Dennen, V. Learner issues with wwwbased systems. En: International Journal of Educational Telecommunications. Vol. 5 N. ${ }^{\circ}$ $4,1999$.

Brown, C. A. From the what and why to the how of course support systems -the value of the teachers' perspective. En: International Journal of Educational Telecommunications. Vol. 5, N. 4, 1999.

Candy, P. C. y Crebert, R. G. Teaching now for learning later: The transfer of learning skills from the academy to the workplace. Papel presentado en el $8^{\text {th }}$ Annual Australiasian Learning and Language Conference Brisbane. July, 1990.

Eggleston, C. Collaborative Technologies-Overview of the State of the Art, 1997.

Ellis, A. y Phelps, S.Staff development for online delivery: A collaborative team-based actionn learning model. En: ASCILITE. Diciembre, 1999.

Jonassen, D. Y Tessmer, M. An outcomes based taxonomy for instructional systems design, evaluation and research. En: Training Research Journal. (2) pp. 11-46, 1997.

Looms. Survey of course and test delivery/ management systems for distance learning. En: http://tangle.seas.gwu.edu/ tlooms/assess.html. Visto el 21de julio de 1999 .

Martín de Hijas, P. La iniciativa ADL SCORM. En: Boletín Learnet. Marzo. http://www. euroforum.cicei.ulpg.es/learnet, 2001.

Robson. WWW-based course-support systems: The firts generation. En: International Journal of Educational Telecommunications. Vol. 5, N. 4, 1999.

Varios autores. Teleformación: un paso más en el camino de la formación continua. Madrid, Fundesco, 1998.
Nachmias, R. y otros. Taxonomy of educational websites - a tool for supporting research, development and implementation of webbased learning. En: International JI of Educational Telecommunications. $5(2), 1999$, 193-210.

Nuñez, A. Comparación del campus virtual de la British Open University y del Campus de Florida State University: Constructivismo vs Conductivismo. En: On Line Educa. Madrid, 2000.

Jornet, J. M. y Suarez J. M. (Coords) . Estudio de perfiles de teleformabilidad. Informes Proyecto TETRA Studio 2000. Valencia, 1999.

Casado, R. El aprovechamiento de las TIC para la creación de redes de aprendizaje colaborativo. En: On Line Educa. Madrid, 2000 .

Pagés Santacana, A. Universidades virtuales: El caso de la Universidad Oberta de Catalunya (UOC) . EDUTEC N. ${ }^{\circ}$ 14. Mayo. http://www. edutec.rediris.es, 2001 .

Vail, K. Online learning grows up. Publicado por Electronic School. http://www.electronicschool.com, 2001.

Goñi, J. J. y Vea, R. Lecciones aprendidas de una comunidad de aprendizaje en Ibermática. En: Esphera. N.॰ 9, año III, 2. Trim., 2002.

Adanes, G. y Gil de Rozas, J. Aprendiendo juntos. En: Esphera. N. ${ }^{\circ}$, año III, 2.॰ Trim., 2002.

Lewis, R. Grupos de trabajo en comunidades virtuales. Publicado en: http://www.uoc.es. Julio, 2001.

Bates, A. W. Theory and practice in the use of technology in distance education in Keegan. En: D. Ed. Theoretical principles of distance education. Londres/Nueva York, Routledge, 1993.

Lara, S. Revisión de software para evaluar formativamente a los alumnos a través de Internet. Publicado en: Quaderns Digitals, 2001 . 